\title{
CL-SA-OFDM: cross-layer and smart antenna based OFDM system performance enhancement
}

\author{
Shivapanchakshari T. G. ${ }^{1}$ H. S. Aravinda ${ }^{2}$ \\ ${ }^{1}$ Department of Electronics and Communication Engineering, Cambridge Institute of Technology, India \\ ${ }^{1}$ Visvesvaraya Technological University, India \\ ${ }^{2}$ Department of Electronics and Communication Engineering, \\ Jagadguru Sri Shivarathreeshwara Academy of Technical Education (JSSATE), India
}

\begin{tabular}{l}
\hline \hline Article Info \\
\hline Article history: \\
Received Sep 25, 2019 \\
Revised Mar 7, 2020 \\
Accepted Mar 21, 2020 \\
\hline
\end{tabular}

Keywords:

Complexity

Fixed bit rate (FBR)

Modulation techniques

Orthogonal frequency division

multiplex (OFDM)

Smart antenna

\begin{abstract}
The growing usage of wireless services is lacking in providing high-speed data communication in recent times. Hence, many of the modulation techniques are evolved to attain these communication needs. The recent researches have widely considered OFDM technology as the prominent modulation mechanism to fulfill the futuristic needs of wireless communication. The OFDM can bring effective usage of resources, bandwidth, and system performance enhancement in collaboration with the smart antenna and resource allocation mechanism (adaptive). However, the usage of adaptive beamforming with the OFDM leads to complication in the design of medium access layer and which causes a problem in adaptive resource allocation mechanism (ARAM). Hence, the proposed manuscript intends to design an OFDM system by considering different switched beam smart antenna (SBSA) along with the cross-layer adaptive resource allocation (CLARA) and hybrid adaptive array (HAA). In this, various smart antenna mechanism are considered to analyze the quality of service (QoS) and complexity reduction in the OFDM system. In this paper, various SA schemes are used as per the quality of service $(\mathrm{QoS})$ requirement of the different users. The performance analysis is conducted by considering data traffic reduction, bit-rate reduction, and average delay.
\end{abstract}

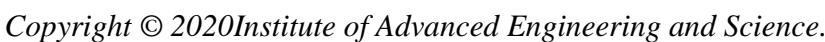
All rights reserved.

\section{Corresponding Author:}

Shivapanchakshari T. G.,

Department of Electronics and Communication Engineering,

Cambridge Institute of Technology,

Krishnarajapura, Bengaluru, Karnataka 560036, India.

Email: tgsresearch2013@gmail.com

\section{INTRODUCTION}

The OFDM system has become a prominent technique in support of modern broadcasting applications [1]. OFDM is a physical layer architecture which uses wireless standards like IEEE802.11a/g, Long Term Evolution (LTE), etc. [2]. The significant advancement in the OFDM is that it can separate the high rated data streams into parallel data streams of the low rate and is processed over different subcarriers simultaneously [3-5]. The use of smart antennas with the OFDM can offer better efficiency in offering higher data communication. The traditional mobile communication systems have adapted smart antennas to overcome the challenges and fulfill requirements of wireless communication [1-8]. Hence, the combination of the smart antenna with OFDM can yield a better communication system with improved spectrum efficiency, channel capacity, coverage range, and performance [9]. The smart antennas can be classified as an adaptive array smart antenna (AASA) and Switched-beam Smart Antenna (SBSA). The significant feature of the AASA is that it tracks each service user within a given frame of the cell by using an alternative adaptive beam pattern. However, the SBSA system tracks every user by collecting the beam patterns based on user location [10-12]. Compared to the SBSA, the AASA can offer better 
performance as it is least complicated and is need to be relay on the proper channel estimation (SE) and convergence speed of the beamforming algorithm [5,6]. Hence, the use of AASA over MAC layer leads to challenges in proper implementation.

This manuscript introduces a design of OFDM system considering switched beam smart antennas (SBSAs) and cross-layer adaptive resource allocation (CLARA) and hybrid adaptive array (HAA). This hybrid smart antennasystem (HSAS) combines both AASA and SBSA for system performance enhancement than the existing system. Algorithm and implementation (section 2), results in the analysis (section 3) and conclusion \& future scope (section 4).

The significant data communication in todays and future wireless connectivity can be attained through better bandwidth as well as transmission rate. Becauase of limited available bandwidth, achieving higher data communication is a critical task. Hence, an adaptive mode of resource allocation can be considered. To achieve performance improvement in wireless communication, the combination of the physical layer (Phyl) and media access control (MAC) can be used. OFDM is mainly considered a better communication technique to fulfill the upcoming data communication needs (Rohling et al. [13]). The OFDM is widespread in many of the architectures such as IEEE $802.11 \mathrm{~g}$, long term evolution (LTE), etc. (Hu et al. [14]). The feature of OFDM is that it separates the high rate streams of data into low rate streams of data over and simultaneously processed over the sub-carrier [14]. In recent times various research tries were focused on attaing effective data communication. The prior work of (Shivapanchakshari and Aravinda [15]) has provided the research ideas of improving the system performance of the smart antenna.

Further, [16] a switched beam smart antenna is introduced to enhance the channel efficiency as well as OFDM system performance. A roubstmeachanism of beamforming (Space-time) is introduced in (Zhang and Wang [17]) for suppressing the multiuser interference. A subspace analysis is performed to train the data blocks and constraints corresponding to the signal subspace. The outcomes of the [17] have suggested that the space time beamforming approach is more significant for time domain equalizer than existing techniques. The work of (Subramanium and Rout [18]) presented an artificial intelligence (AI) absed turbo coded OFDM model to enhance the performance of the bit error rate (BER) which is a prime factor for network evaluation. The model comes up with improved BER performance and reduced delay. The combined work of (Benzarti and Abdellaoui [19]) introduced the synchronization model for SISO amd MIMO OFDM systems by adapting smart antenna technolog and attained better frequency synchronization. In (Boud et al. [20]) an angle of arrival and parametric method is presented for the estimation of Doppler frequency. The model introduces an aeronautical satellite channel for estination. The model reduces the impact of inter carrier interference in the aeronautical satellite environment. Concept of massive MIMO-OFDM for the uplink system is presented in (Jacobssson et al. [21]). The Bussgang theorem is introduced to impair the hardware for multiuser MIMO-OFDM. The outcomes suggest that it reduces the distortions caused by the non-ideal hardware. The fading characteristics reduction in wireless communication among vehicle to vehicle is presented in (Kim et al. [22]) by using smart antennas. With this mechanism, the fading effect is reduced.

The performance analysis of MIMO-OFDM physical layer in the WiMAX with low-density parity-check (LDPC) is performed in (Cheema and Kulkarni [23]) under various modulation mechanism. The high capacity at low BER is achieved with the use of LDFC codes. These graphs are referred to as the Tanner graph having short cycles which minimizes the LDFC decoder's performance. The outcomes suggest that the proposed MIMO-OFDM improved the transmission rate, transmission reliability, and transmission range. A deep learning based archirecture for OFDM is introduced in (Balevi and Andrews [24]).

Under complex single bit quantization constraint, the complexity, accuracy is reduced and leads to complex data detection. The proposed design lowers the BER at SNR of dB. The implementation of the MIMO-OFDM in SATCOM is discussed in (Suganya and Gandhiraj [25]). The compressive survey discusses the diffeent MIMO-OFDM techniques for satellite communication and idealizes the upcoming research scopes. The work of (Tao and Zhou [26]) discussed the indoor localization mechanism for MIMO with diversity property of smart antenna and achieved a high level of accuracy in the multipath environment. The comparative analysis of Seydnejad and Akhzari [27]) compared Pre-FFT and post FFT based time and frequency domain beamforming methods, respectively, for one-input multiple-output (OIMO) OFDM systems.

Similarly, the (Chen et al. [28]) have discussed future ideas for wireless communication and challenges in it. A recent work of (Boustani et al. [29]) introduces the direction of arrival algorithm for the smart antenna to analze the performance in wireless communication. The work of (Rozaini et al. [30]) proposed the concept of PAPR reduction in OFDM system and attained low complex system. Another work (Ait-Saadi et al. [31]) introduced the self-adaptive algoritjm for PAPR reduction in OFDM system. From the research survey, it has been observed that some of the problems are yet to be addressed in the research domain of Smart antenna and OFDM. The work of Youssif et al. [32] have discussed residue 
number method as coding methof for digital image brodcasting on MIMO-OFDM transceiver broadcating method. The ouctome of this study illustrates about performance of the broadcasting model with as well as without RNM-coding execution. Youssef et al. [33] have demonstrated a residue numbers as a coding method has been impeded in the MIMI-OFDM techniques, due to its compassion of the OFDM to offsets that lead to loss of orthogonally, intrusion and performance poverty. The work done by Leftah and Alminshid [34] demonstrates the DHT precoded MIMO-OFDM method on frequency-chosen fading model with huge-size quadrature modulation. The outcome of this research study demonstrates that the communication performance and channel capability of the proposed methods which is superior to traditional MIMO-OFDM exclusive of a pre-coder. The problem statement of the manuscript is given below.

The consideration of the smart antenna with OFDM can improve the throughput and by which the reuse of the same subcarriers can be possible over the various beams for spatially separated users [1-15]. In order to enhance the user signal-to-interference-plus-noise ratio (SINR) as it uses higher modulation schemes. The OFDM system without adaptive resource allocation and with AASA can offer improved performance and lower cost than the implementation of SBSA [8]. If adaptive resource allocation is used with AASA at base station requires covering each user and is a not better idea because: (a) beamforming of AASA depends on the symbols training or Direction of Arrival (DOA) whereDOA put lots of load on the physical layer and MAC layers. The users in a cell are of low data rate (LDR) or voice users. The LDR holds only one or two subcarriers. If the system is fully loaded, then the LDR users increases than the separate adaptivebeamformingbecause the implementation process needs to be performed separately for different users [10]. Hence, to have the support towards the AASA in the OFDM system, the adaptive resource allocation can lead to complicated optimization. Hence, the system becomes unstable, which means the adaptive algorithm cannot achieve convergence [11, 12]. Thus, a suboptimal and realistic smart antenna mechanism is needed to implement in the OFDM with adaptive resource allocation. The research method adopted is given below.

The manuscript aims to explore the significant factors of the smart antenna usage over OFDM resource allocation (an adaptive mechanism). The architectural flow of the model is presented in Figure 1 and is named as "Cross-layer and smart antenna based OFDM (CL-SA-OFDM) system." The proposed model utilizes different processing units. In this, a different set of users $1,2 \ldots \mathrm{N}$ with serial bit streams are given to the subcarriers and bit allocation unit (BAU). In BAU, user bits are loaded on different subcarriers. Later, the BAU data streams outputs are considered for the Q-adaptive modulator and to the modulation levels appropriate modulation (appropriate mod.) are chosen for different subcarriers. Then, the outputs of adaptive modulators are considered for beamforming module in which smart antenna mechanisms are needed to be considered to attain user experience in Quality of Service (QoS). AASA can be used to perform the real video data transmission (of high data rate) of subcarriers group. Also, for low data (such as voice data) processing, AASAutilizes less subcarriers. The adaptive resource allocation module consists of a hybrid smart antenna which helps in controlling the process processing. The data is converted samples of time-domain samples by using inverse FFT (IFFT) and to this Cyclic Prefix (CP) is added. Then the transmitted data streams will be transferred over via antennas $(1,2 \ldots \mathrm{N})$.

\section{ALGORITHM IMPLEMENTATION}

The algorithm to perform the significant allocation of resources by using adaptive mechanism at closs layer and smart antenna based OFDM system and achieve better performance in terms of complexity. The description of the algorithm is given below.

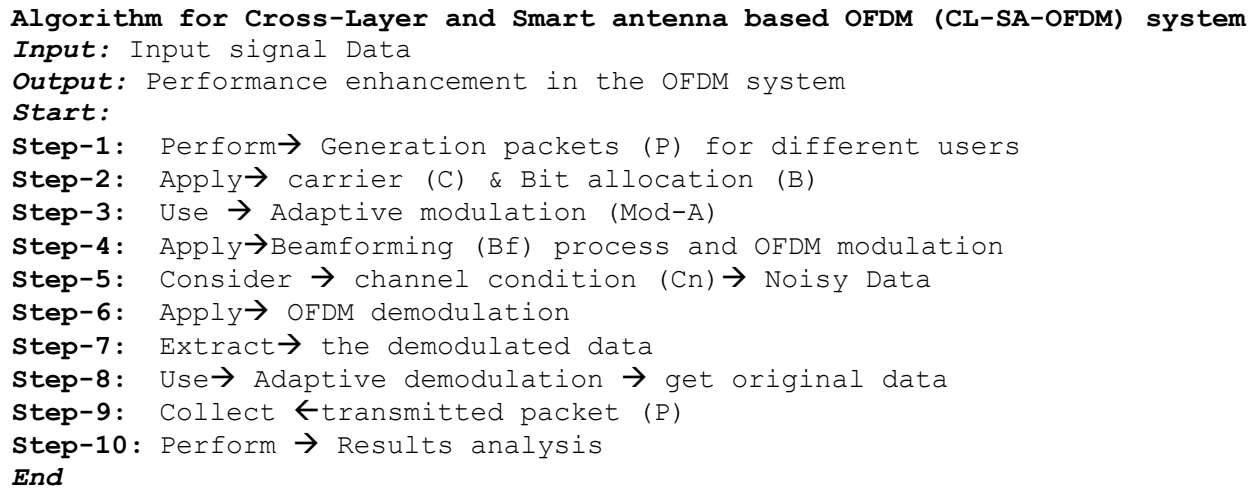


The data acquired from the users are utilized to perform the generation of packets $(\mathrm{P})$. On these generated $\mathrm{P}$, the carrier $(\mathrm{C})$ and Bit Allocation $(\mathrm{B})$ is applied. The adaptive modulation $(\operatorname{modA})$ is used which modifies the transmission channel characteristics, and it can be used in mobile communication. Then Beamforming (Bf) is utilized and is an access point considers more antennas to transfer the same signal. Then the Channel condition $(\mathrm{Cn})$ is applied to get the noisy data over OFDM modulated data. OFDM demodulation is applied at the receiver side and obtain the transmitted $\mathrm{P}$, original data. Finally, the performance analysis is conducted on the generated results.

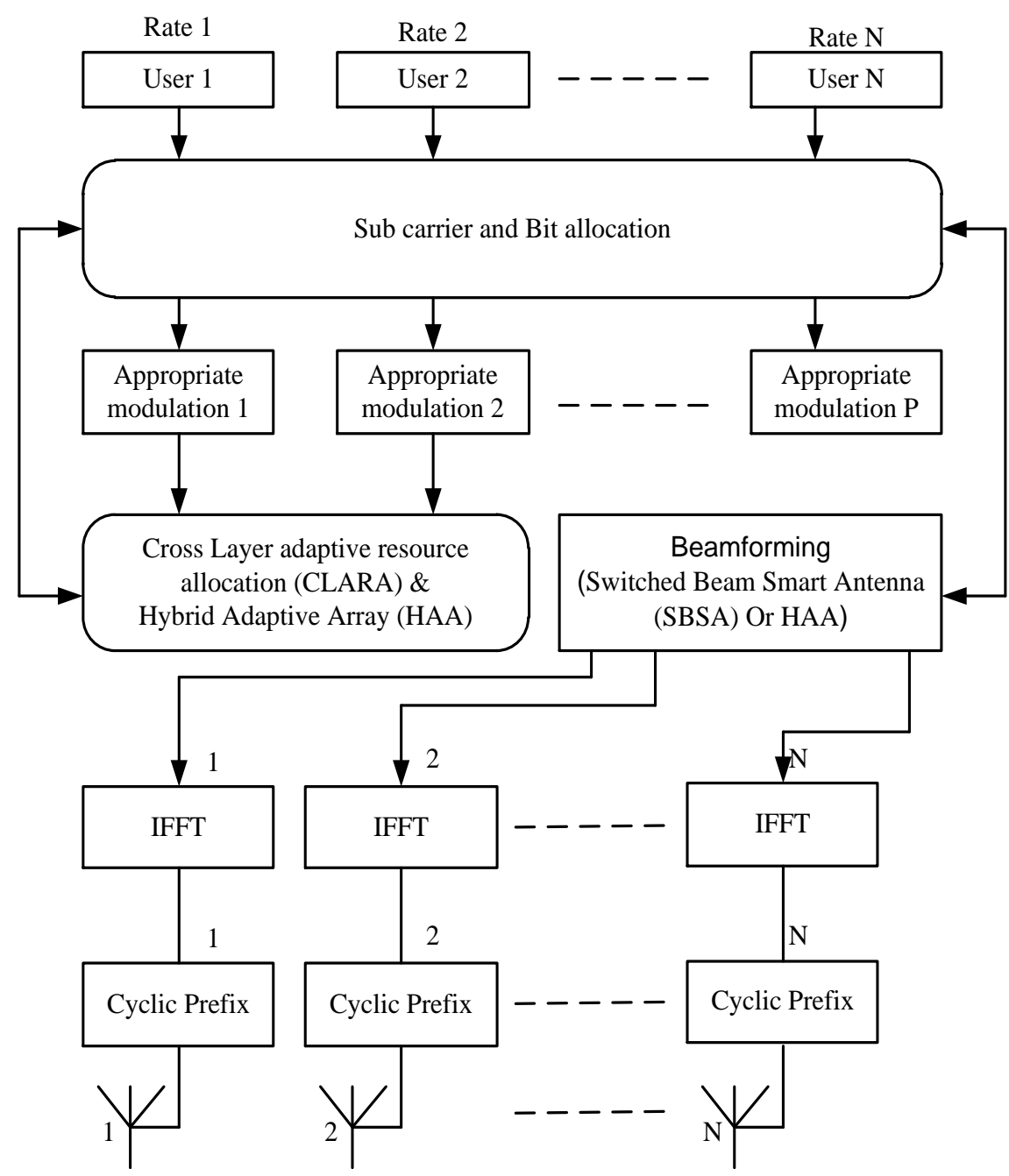

Figure 1. Architectural diagram of the proposed system

\section{RESULTS ANALYSIS}

The design of the Cross-Layer and Smart antenna based OFDM system (CL-SM-OFDM) is performed over MATLAB. While designing the OFDM system, the III beams and VI beams antenna methods are considered. The SAs like Hybrid SA, Perfect SA, and Switched beam SA are considered for performance analysis under random (35 to 210) number of users. Figure 2 represents the generated input binary data stream, where the random data bits are considered as inputs for OFDM and are need to be transmitted.

The carrier power of the binary data is represented in Figure 3 with a power level of 0:0.2:1 for each bit to the carrier, which indicates the resource allocation. Figure 4 represents the outcome of the adaptive modulation applied to the binary data which modifies the transmission channel characteristics, and it can be used in mobile communication. The beamforming of the proposed system is represented in Figure 5, which is a radio frequency (RF) management and is an access point considers more antennas to transfer the same signal. 
The OFDM modulation is used to modulate the previous modulated data into another signal having higher bandwidth and frequency. The output of the OFDM modulated data is given in Figure 6. Further, channel condition is applied to this OFDM modulated data which gives noisy data as shown in Figure 7. The OFDM demodulated data as shown in Figure 8 is obtained from demultiplexing the subcarrier time-domain signal by using FFT operation in parallel data streams, and it gives the collected signal as shown in Figure 9. Similarly, adaptive demodulation is applied to get the original data as shown in Figure 10.

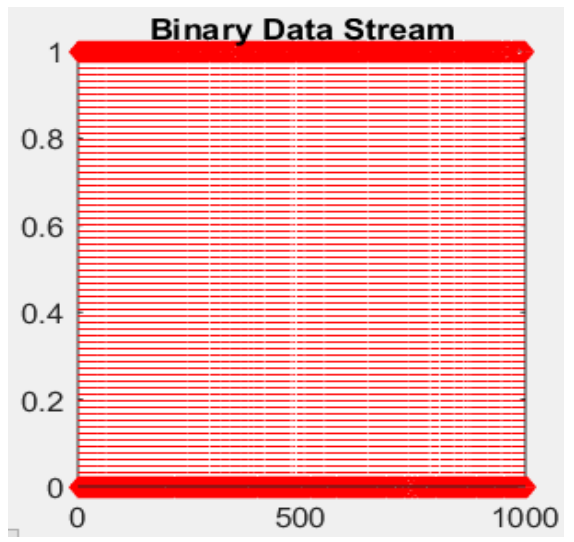

Figure 2. Generated binary data stream

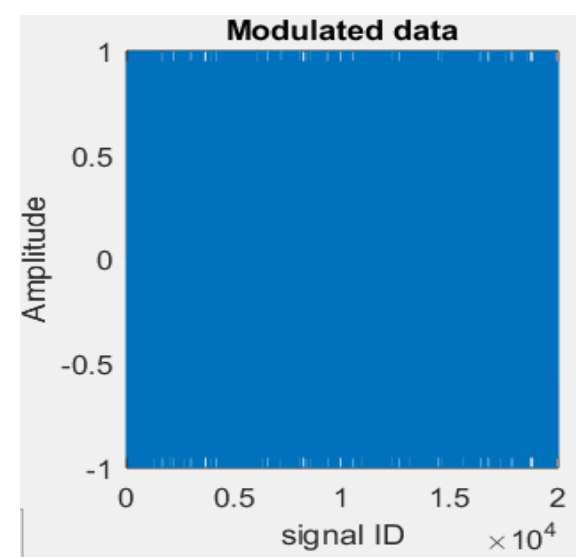

Figure 4. Adaptive modulated data

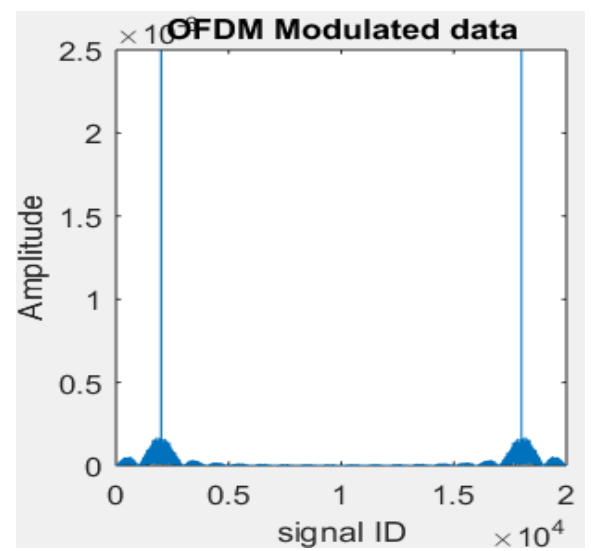

Figure 6. OFDM modulated data

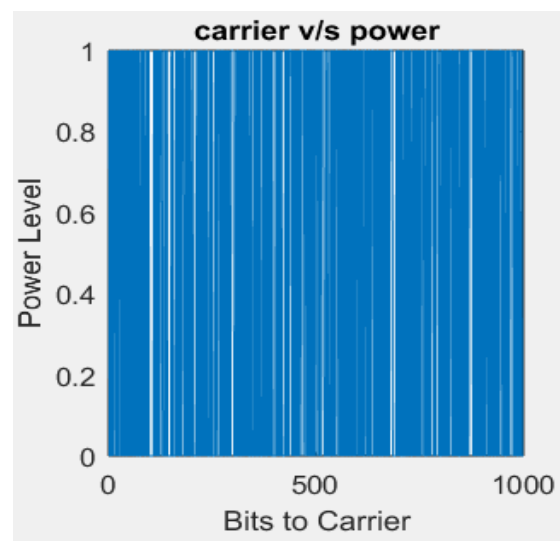

Figure 3. Carrier power level

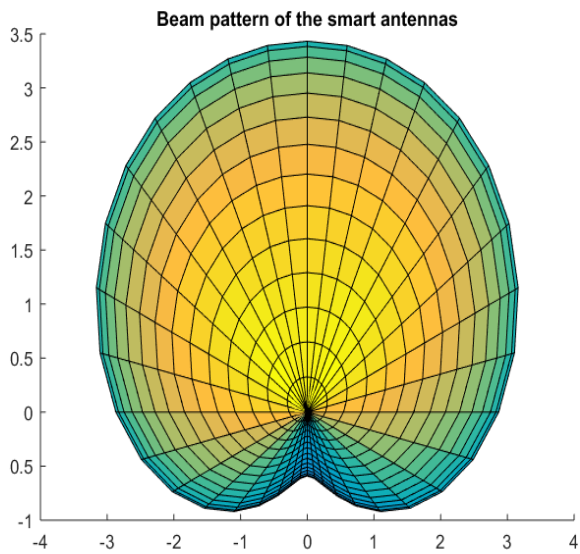

Figure 5. Beamforming

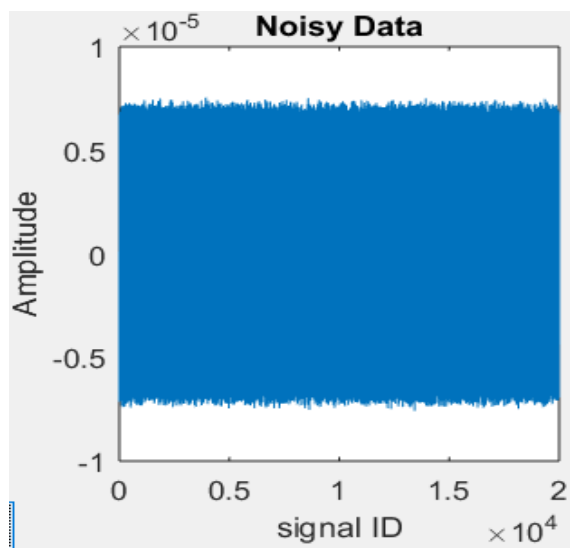

Figure 7. Noisy data 


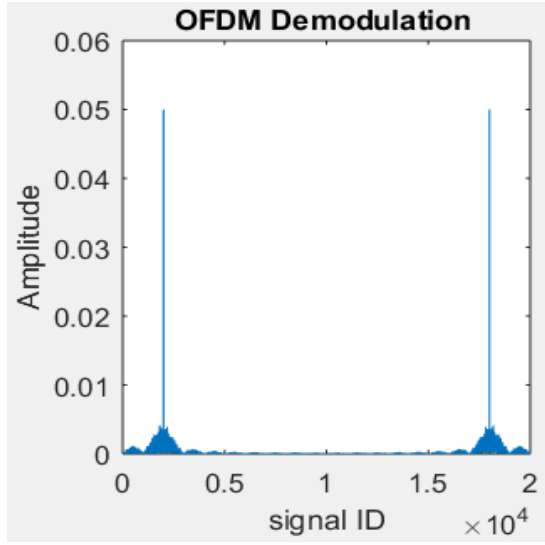

Figure 8 OFDM demodulated data

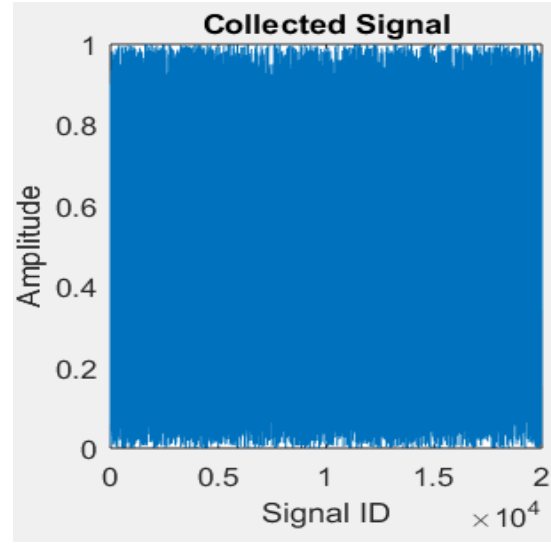

Figure 9. Collected data after OFDM demodulation

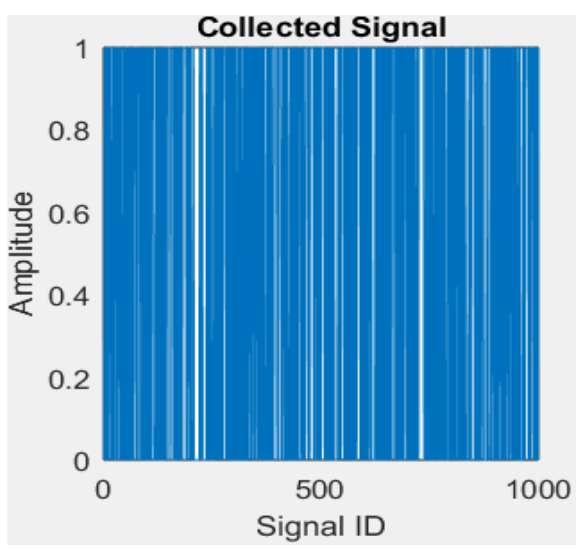

Figure 10. Collected data after Adaptive demodulation

\section{PERFORMANCE ANALYSIS}

The performance analysis of the proposed system is performed with Tables 1-3 by considering voice user packet data traffic degradation analysis, fixed bit-rate degradation analysis and average delay analysis for Method III and Method VI Switched beam smart antenna, Switched Hybrid smart antenna and respectively. From the Tables 1-3 indicates that the perfect smart antenna offers significant performance than the hybrid smart antenna and Switched smart beam antenna. This happens due to the consideration of Switched beam in the Hybrid smart antenna and Switched smart beam antenna while the perfect smart antenna uses voice user packet data traffic, fixed bit rate terminals. The use of AASA is meant to fixed bit rate terminals in Hybrid Smart Antenna. By observing Tables 1-3 voice user packet data traffic degradation (0.00656) analysis, fixed bit-rate degradation (0.0046), and average delay of (5.37). By this, it suggests that system complexity is reduced and performance is enhanced.

Table 1. Voice user data packet degradation analysis from method III and method VI switched smart beam antenna

\begin{tabular}{ccc}
\hline Methods/Users & $\begin{array}{c}\text { Voice data traffic reduction } \\
\text { Method III }\end{array}$ & Method VI \\
\hline 35 & 0.003165 & 0.002354 \\
65 & 0.005907 & 0.004896 \\
95 & 0.009249 & 0.006316 \\
125 & 0.012864 & 0.008337 \\
155 & 0.015666 & 0.011358 \\
185 & 0.018782 & 0.013471 \\
\hline
\end{tabular}

Int J Elec \& Comp Eng, Vol. 10, No. 5, October 2020 : 4663 - 4670
Table 2. Fixed bit rate degradation analysis from method III and method VI switched hybrid smart antenna

\begin{tabular}{ccc}
\hline \multirow{2}{*}{ Methods/Users } & \multicolumn{2}{c}{ The fixed bit-rate reduction } \\
Method III & Method VI \\
\hline 35 & 0.002911 & 0.002570 \\
65 & 0.005859 & 0.004474 \\
95 & 0.008544 & 0.006712 \\
125 & 0.011409 & 0.008282 \\
155 & 0.013896 & 0.010342 \\
185 & 0.017143 & 0.012720 \\
\hline
\end{tabular}


Table 3. Average delay analysis from method III and method VI perfect smart antenna

\begin{tabular}{ccc}
\hline Methods/Users & \multicolumn{2}{c}{ Average delay analysis } \\
& Method III & Method VI \\
\hline 35 & 2.323720 & 2.051754 \\
65 & 4.240600 & 3.292697 \\
95 & 7.645894 & 4.945828 \\
125 & 9.260928 & 6.448050 \\
155 & 11.533689 & 8.371088 \\
185 & 14.879313 & 9.688584 \\
\hline
\end{tabular}

\section{CONCLUSION}

The manuscript considered the concerns of the high-speed data communication and proposed an efficient cross-layer adaptive resource allocation (CLARA) along with the HAA and SBSA for OFDM systems. In this, different smart antenna schemes are considered to analyze the performance as per the user QoS requirement. The schemes include perfect smart antenna, Switched smart beam antenna, and Hybrid smart antenna with III or VI beam antennas along with adaptive resource allocation (ARA). For experimentation, the number of users is randomly selected. The outcomes are analyzed with respect to the traffic of voice user, fixed bit rate (FBR), and Average Delay by which the performance is enhanced with least complexity. The scope of the proposed manuscript is that it can be considered with other beam antennas at different traffic condition and security concerns of the OFDM system can also be used as a performance metric.

\section{REFERENCES}

[1] R. Gerzaguet, et al., "5G multi-service field trials with bf-ofdm," in 2017 IEEE Globecom Workshops (GC Wkshps), 2017.

[2] J. Muñoz, et al., "Overview of IEEE802. $15.4 \mathrm{~g}$ OFDM and its Applicability to Smart Building Applications," in 2018 Wireless Days (WD), 2018.

[3] C. Studer and G. Durisi, "Quantized massive mu-mimo-ofdm uplink," IEEE Transactions on Communications, vol. 64, no. 6, pp. 2387-2399, 2016.

[4] S. Jacobsson, et al., "Linear precoding with low-resolution DACs for massive MU-MIMO-OFDM downlink," IEEE Transactions on Wireless Communications, vol. 18, no. 3, pp. 1595-1609, 2019.

[5] S. Jacobsson, et al., "Nonlinear precoding for phase-quantized constant-envelope massive MU-MIMO-OFDM," in 2018 25th International Conference on Telecommunications (ICT), 2018.

[6] S. Buzzi, et al., "Single-carrier modulation versus OFDM for millimeter-wave wireless MIMO," IEEE Transactions on Communications, vol. 66, no. 3, pp. 1335-1348, 2017.

[7] E. Balevi, and J. G. Andrews, "One-bit OFDM receivers via deep learning," IEEE Transactions on Communications, 2019.

[8] S. Gökceli, et al., "Implementation of pre-FFT beamforming in MIMO-OFDM," in 2015 9th International Conference on Electrical and Electronics Engineering (ELECO), 2015.

[9] C. Gu, et al., "Compact smart antenna with electronic beam-switching and reconfigurable polarizations," IEEE Transactions on Antennas and Propagation, vol. 63, no. 12, pp. 5325-5333, 2015.

[10] A. S. Oluwole, and V. M. Srivastava, "Analysis of smart antenna with improved signal quality and spatial processing," in 2016 Progress in Electromagnetic Research Symposium (PIERS), 2016.

[11] C. Kim, T. Kim, and J.-Y.Seol, "Multi-beam transmission diversity with hybrid beamforming for MIMO-OFDM systems," in 2013 IEEE Globecom Workshops (GC Wkshps), 2013.

[12] M. Wu, et al., "On OFDM and SC-FDE transmissions in millimeter wave channels with beamforming," in 2016 IEEE 83rd Vehicular Technology Conference (VTC Spring), 2016.

[13] H. Rohling and T. May, "Comparison of PSK and DPSK Modulation in a Coded OFDM System," IEEE 47th Vehicular Technology Conference, vol. 2, 1997.

[14] H. Hu, K. Guo, and M. Weckerle, "Hybrid smart antennas for OFDM systems-a cross-layer approach," IEEE 16th International Symposium onPersonal, Indoor and Mobile Radio Communications, 2005 - PIMRC 2005, vol. 4, 2005.

[15] T. G. Shviapanchakshari and H. S. Aravinda, "Review of Research Techniques to Improve System Performance of Smart Antenna," Open Journal of Antennas and Propagation, vol. 5, no. 02, pp. 83, 2017.

[16] T. G. Shviapanchakshari and H. S. Aravinda, "An Efficient Mechanism to Improve the Complexity and System Performance in OFDM Using Switched Beam Smart Antenna (SSA)," in Computer Science On-line Conference, 2019. doi: 10.1007/978-3-030-19813-8_7.

[17] W. Zhang, et al., "Robust multi-branch space-time beamforming for OFDM system with interference," Digital Signal Processing, vol. 65, pp. 63-70, 2017.

[18] P. Subramanium, and R. D. Raut, "AI-enabled turbo-coded OFDM system for improved BER performance," The Journal of Supercomputing, pp. 1-11, 2018. 
[19] M. Benzarti and Z.Abdellaoui, "ComparatVIe Study of Frequency Synchronization in SISO and MIMO-OFDM Systems," in 2016 7th International Conference on Sciences of Electronics, Technologies of Information and Telecommunications (SETIT), 2016.

[20] H. Boud, et al., "Doppler estimation for aeronautical satellite OFDM system," in 2017 8th IEEE Annual Information Technology, Electronics and Mobile Communication Conference (IEMCON), 2017.

[21] S. Jacobsson, et al., "MassVIe MU-MIMO-OFDM Uplink with Hardware Impairments: Modeling and Analysis," in 2018 52nd Asilomar Conference on Signals, Systems, and Computers, 2018.

[22] J.-H. Kim, et al., "Performance analysis of fading reduction using the dVIersity antenna and $2 \times 2$ smart antenna for 802.11 p WAVE V2V communication," in 2017 Ninth International Conference on Ubiquitous and Future Networks (ICUFN), 2017.

[23] M. Cheemaand S. A. Kulkarni, "Analysis of MIMO OFDM Based WiMAX system with LDPC," in 2015 Fifth International Conference on Advances in Computing and Communications (ICACC), 2015.

[24] E. Balevi, and J. G. Andrews, "Reliable Low Resolution OFDM ReceVIers via Deep Learning," in 2018 52nd Asilomar Conference on Signals, Systems, and Computers, 2018.

[25] M. Suganya, and R. Gandhiraj, "Applicability of MIMO and OFDM technology to SATCOM," in 2016 International Conference on Communication and Signal Processing (ICCSP), 2016.

[26] C. Tao and B. Zhou, "Indoor Localization with Smart Antenna System: Multipath Mitigation with MIMO Beamforming Scheme," in 2017 IEEE 14th International Conference on Mobile Ad Hoc and Sensor Systems (MASS), 2017.

[27] S. R. Seydnejad, and S.Akhzari, "Performance evaluation of pre-and post-FFT beamforming methods in pilot-assisted SIMO-OFDM systems," Telecommunication Systems, vol. 61, no. 3, pp. 471-487, 2016.

[28] D. Chen, et al., "OQAM-OFDM for Wireless Communications in Future Internet of Things: A Survey on Key Technologies and Challenges," IEEE Internet of Things Journal, vol. 5, no. 5, pp. 3788-3809, 2018.

[29] B. Boustani, et al., "Performance analysis of direction of arrival algorithms for smart antenna," International Journal of Electrical and Computer Engineering (IJECE), vol. 9, no. 6, pp. 4873-4881, 2019.

[30] M. D. Rozaini, et al., "Median codeword shift (MCS) technique for PAPR reduction with low complexity in OFDM system," International Journal of Electrical and Computer Engineering (IJECE), vol. 9, no. 6, pp. 4882-4888, 2019.

[31] H. Ait-Saadi, J.-Y.Chouinard, and A.Guessoum, "A PAPR Reduction for OFDM Signals Based on Self-Adaptive Multipopulation DE algorithm," International Journal of Electrical and Computer Engineering (IJECE), vol. 7, no. 5, pp. 2651-2660, 2017

[32] M. I. Youssef, A. E. Emam, and M. A. Elghany, "Image multiplexing using residue number system coding over MIMO-OFDM communication system," International Journal of Electrical and Computer Engineering (IJECE), vol. 9, no. 6, pp. 4815-4825, 2019.

[33] M. I. Youssef, and A. E. Emam, "ICI and PAPR enhancement in MIMO-OFDM system using RNS coding," IOP Conference Series: Materials Science and Engineering, vol. 610, no. 1, 2019.

[34] H. A. Leftah and H. N. Alminshid, "Channel capacity and performance evaluation of precoded MIMO-OFDM system with large-size constellation," International Journal of Electrical and Computer Engineering (IJECE), vol. 9, no. 6, pp. 5024-5030, 2019.

\section{BIOGRAPHIES OF AUTHORS}

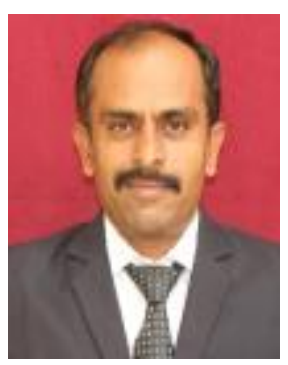

Shivapanchakshari T. G. is working asassistant professor in Cambridge Institute of Technology, Bangalore. He is pursuing his Ph.D. degree in Visvesvaraya Technological University, Belagavi, India. His fields of interest are smart antennas, electromagnetics and digital communication. He has published many papers on smart antennas.

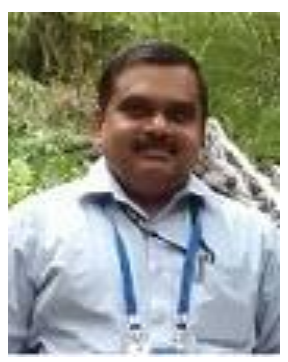

Dr. H. S. Aravinda is a professor and Head of the department of ECE in JSSATE, Bangalore. He has obtained his Ph D degree from Visvesvaraya Technological University, Belagavi, India. His fields of interest are Signal Processing and Communication. He has published many papers in reputed journals in the field of signal processing. Presently, he is guiding six research scholars. 